\title{
COMMUTATOR HOPF SUBALGEBRAS AND IRREDUCIBLE REPRESENTATIONS
}

\author{
EDWARD S. LETZTER \\ letzter@temple.edu
}

\begin{abstract}
S. Montgomery and S. Witherspoon proved that upper and lower semisolvable, semisimple, finite dimensional Hopf algebras are of Frobenius type when their dimensions are not divisible by the characteristic of the base field. In this note we show that a finite dimensional, semisimple, lower solvable Hopf algebra is always of Frobenius type, in arbitrary characteristic.
\end{abstract}

\section{INTRODUCTION}

1.1. Kaplansky conjectured in [6] that a finite dimensional semisimple Hopf algebra $H$ over an algebraically closed field $k$ must be of Frobenius type (i.e., the dimensions of the irreducible representations of $H$ must divide $\operatorname{dim} H$ ). Montgomery and Witherspoon established Kaplansky's conjecture when $H$ is upper or lower semisolvable (cf. [11, §3]), provided $\operatorname{dim} H$ is not divisible by the characteristic of $k$ [11]. Etingof and Gelaki proved that $H$ is of Frobenius type when it is quasitriangular, provided either $k$ has characteristic zero [5] or $H$ is cosemisimple [4].

1.2. Continue, throughout this note, to let $k$ be an algebraically closed field of arbitrary characteristic. Consider a series of Hopf algebras

$$
k=H_{0} \subset H_{1} \subset \cdots \subset H_{t-1} \subset H_{t}=H,
$$

such that $H_{i-1}$ is normal in $H_{i}$, and such that $H_{i} / H_{i} H_{i-1}^{+}$is commutative, for all $1 \leq i \leq t$. (Normality is defined, e.g., in [10,3.4.1], and $H_{i}^{+}$denotes the kernel of the augmentation map.) In $[11, \S 3], H$ is said to be lower solvable. Lower solvable Hopf algebras are obvious generalizations of group algebras of solvable groups, and we can view each $H_{i-1}$ as a "commutator Hopf subalgebra" of $H_{i}$.

In this note we prove:

This research was supported in part by a grant from the National Security Agency. 
1.3 Theorem. Let $H$ be a finite dimensional, semisimple, lower solvable $k$-Hopf algebra. Then $H$ is of Frobenius type.

1.4. When the dimension of $H$ is not divisible by the characteristic of $k$, the preceding result follows from the above cited work of Montgomery and Witherspoon [11, 3.4]. Of course, when $H$ is quasitriangular, and when either $k$ has characteristic zero or $H$ is cosemisimple, the result follows from the above cited work of Etingof and Gelaki [4; 5]. We will see in $\S 4$ that Theorem 1.3 applies to cases not already covered in this earlier work.

1.5. Our proof of Theorem 1.3 is based on the approach found in $[8 ; 9]$.

1.6 Notation. (i) Vector spaces, tensor products, algebras, Hopf algebras, representations, and homomorphisms are over $k$ unless otherwise noted.

(ii) We use $\Delta$ for coproducts, $\epsilon$ for counits, and $S$ for antipodes. Given an element $h$ in a Hopf algebra $H$, we set $\Delta h=\sum h_{1} \otimes h_{2}$. Given a $k$-algebra homomorphism $\chi: H \rightarrow k$, the 1-dimensional module defined by $h . v=\chi(h) v$ for $h \in H$ and $v \in k$ will be denoted $k_{\chi}$.

Acknowledgement. My thanks to R. Guralnick and D. Passman for helpful explanatory comments regarding (2.7). My thanks to the referee for several helpful remarks and in particular for pointing me toward the examples discussed in $\S 4$.

\section{Commutator Hopf Subalgebras}

This section provides the lemmas necessary to prove Theorem 1.3.

2.1 Assumptions. In this section we let $H$ and $K$ be (not necessarily finite dimensional) $k$-Hopf algebras satisfying the following properties: (1) $K$ is a normal Hopf subalgebra of $H$, (2) every finite dimensional left or right simple $H$-module is semisimple as a $K$-module, (3) every finite dimensional irreducible representation of $H / H K^{+}$is 1-dimensional. (Recall that $H K^{+}=K^{+} H$ is a Hopf ideal of $H$, because $K$ is a normal Hopf subalgebra of $H$; see $[10,3.4 .2]$.

2.2 Example. Let $G$ be a (not necessarily finite) group with its commutator subgroup $G^{\prime}$ having finite index. Setting $H=k G$ and $K=k G^{\prime}$, we can see as follows that the hypotheses of (2.1) are satisfied: To start, $K$ is a normal Hopf subalgebra of $H$ because $G^{\prime}$ is a normal subgroup of $G$. Next, $H / H K^{+}$is commutative and finite dimensional, and so all of its irreducible representations are 1-dimensional. Lastly, it follows from Clifford Theory that every left or right simple $H$-module is semisimple as a $K$-module; see, for example, [12, 8.1.3].

2.3. Let $A$ be a subring of a ring $B$. Recall that a prime ideal $P$ of $B$ lies over a prime ideal $Q$ of $A$ provided $Q$ is minimal over $P \cap A$ (i.e., there are no prime ideals of $A$ that both contain $P \cap A$ and are properly contained in $Q)$. 
2.4 Lemma. Let $P$ be a finite codimensional primitive ideal of $H$ lying over a (necessarily finite codimensional) primitive ideal $Q$ of $K$. Then $H /(P+H Q)$ is a nonzero $H-K$ bimodule (necessarily with left annihilator $P$ and right annihilator $Q)$, and $H /(P+Q H)$ is a nonzero $K-H$-bimodule (necessarily with right annihilator $P$ and left annihilator $Q$ ).

Proof. It follows from assumption 2.1(2) that $K / P \cap K$ is a semisimple $k$-algebra. Therefore, since $Q$ is minimal over $P \cap K$, we see that $Q . X=0$ for some nonzero ideal $X$ of $K / P \cap K$. Hence, $(P+H Q) \cdot X=0$ in $H / P$, and so $P+H Q \neq H$. A similar argument shows that $P+Q H \neq H$.

2.5. (i) Let $\chi: H \rightarrow k$ be a $k$-algebra homomorphism. We will use $\theta_{\chi}$ to denote the $k$-algebra automorphism of $H$ obtained by setting

$$
\theta_{\chi}(h)=\sum \chi\left(h_{1}\right) h_{2}
$$

for $h \in H$. (Note that $\theta_{\chi}$ is bijective because it has inverse $\theta_{\chi^{-1}}$, where $\chi^{-1}$ is the convolution inverse of $\chi$.)

(ii) Let $\alpha$ be a $k$-algebra automorphism of $H$, and let $V$ be a left $H$-module. We will use ${ }^{\alpha} V$ to denote the left $H$-module with action $h * v=\alpha(h) \cdot v$, where "." denotes the original action on $V$. Observe, if $\chi: H \rightarrow k$ is a $k$-algebra homomorphism, that

$$
{ }^{\theta_{\chi}} V \cong k_{\chi} \otimes V,
$$

where the usual tensor-product $H$-module action is employed.

The proof of the next proposition follows arguments found in $[8 ; 9]$.

2.6 Proposition. Let $P_{1}$ and $P_{2}$ be finite codimensional primitive ideals of $H$ both lying over a primitive ideal $Q$ of $K$. Let $V_{1}$ be a simple left $H$-module with annihilator $P_{1}$, and let $V_{2}$ be a simple left $H$-module with annihilator $P_{2}$. Then there exists a $k$-algebra homomorphism $\chi: H \rightarrow k$ such that:

(i) $P_{2}=\theta_{\chi}\left(P_{1}\right)$.

(ii) $V_{1} \cong k_{\chi} \otimes V_{2}$.

Proof. (i) By (2.4) there exists a finite dimensional $H$-K-bimodule factor $M_{1}$ of $H$ with left annihilator $P_{1}$ and right annihilator $Q$. Similarly, there exists a finite dimensional $K$-H-bimodule factor $M_{2}$ of $H$ with left annihilator $Q$ and right annihilator $M_{2}$. Note that $M_{1}$ is naturally a nonzero semisimple (and so projective) right $K / Q$-module and that $M_{2}$ is naturally a nonzero semisimple left $K / Q$-module. Consequently, $M:=M_{1} \otimes_{K} M_{2}$ is a nonzero finite dimensional $H$-H-bimodule factor of $H \otimes_{K} H$. The only possible left annihilator of $M$ in $H$ is $P_{1}$, and the only possible right annihilator of $M$ in $H$ is $P_{2}$. Furthermore, the only possible left annihilator in $H$ of any nonzero left $H$-submodule of $M$ is $P_{1}$, and the only possible right annihilator in $H$ of any nonzero right $H$-submodule 
of $M$ is $P_{2}$. Let $g$ denote the image in $M$ of $1 \otimes 1 \in H \otimes_{K} H$. Since $g$ generates $M$ as an $H$ - $H$-bimodule, it follows that $g \neq 0$.

Next, observe that $M$ is a left $H$-module via

$$
h * m=\sum h_{1} \cdot m \cdot S\left(h_{2}\right)
$$

for $h \in H$ and $m \in M$. Moreover, $\mathrm{kg}$ is a 1-dimensional $K$-submodule under $*$, isomorphic to $k_{\epsilon}$. So consider $H * g$. Observe that it is a finite dimensional $H$-module factor of $L:=H \otimes_{K} k_{\epsilon}$. However, $\left(H K^{+}\right) \cdot L=0$, and we have assumed that every finite dimensional irreducible representation of $H / H K^{+}$is 1-dimensional. Therefore, there exists a nonzero element $e$ of $M$ such that $k e$ is an $H$-module under the $*$-action.

We obtain a $k$-algebra homomorphism $\chi: H \rightarrow k$ such that

$$
h * e=\sum h_{1} \cdot e \cdot S\left(h_{2}\right)=\chi(h) e,
$$

for all $h \in H$.

Notice, for $h \in H$, that

$$
\begin{aligned}
h . e=\sum h_{1} \epsilon\left(h_{2}\right) \cdot e= & \sum h_{1} \cdot e \epsilon\left(h_{2}\right)=\sum h_{1} \cdot e \cdot S\left(h_{2}\right) h_{3} \\
& =\sum\left(h_{1} * e\right) \cdot h_{2}=\sum e \cdot \chi\left(h_{1}\right) h_{2}=e \cdot \theta_{\chi}(h) .
\end{aligned}
$$

Since $0=P_{1} . e=e \cdot \theta_{\chi}\left(P_{1}\right)$, it follows that $\theta_{\chi}\left(P_{1}\right) \subseteq P_{2}$. Since $\theta_{\chi}\left(P_{1}\right)$ is a maximal ideal of $H$, it follows that $\theta_{\chi}\left(P_{1}\right)=P_{2}$. Part (i) follows.

(ii) Set $U$ equal to the left $H$-module ${ }^{\theta_{\chi}} V_{2} \cong k_{\chi} \otimes V_{2}$. By (i), $P_{1} \cdot U=\theta_{\chi}\left(P_{1}\right) \cdot V_{2}=$ $P_{2} . V_{2}=0$. Since $U$ is a simple $H$-module, it must follow that $U \cong V_{1}$.

2.7 Example. The following example, illustrating the applicability of (2.6), served as the original motivation for our work in this note: Set $H=k G$ and $K=k G^{\prime}$, as in (2.2). For each $g \in G$, let $\alpha_{g}$ be the $k$-algebra automorphism of $H$ mapping $h \in H$ to $g^{-1} h g$. Since $G^{\prime}$ is normal in $G$, each $\alpha_{g}$ restricts to an automorphism of $K$ that we will also denote by $\alpha_{g}$.

Now let $U$ be an irreducible finite dimensional left $K$-module, and let $Q$ be the annihilator of $U$ in $K$. Set $M=H \otimes_{K} U$. Note, for each $g \in G$, that the $K$-submodule $g \otimes U$ of $M$ is isomorphic to ${ }^{\alpha_{g}} U$ and so has annihilator $\alpha_{g^{-1}}(Q)$ in $K$. Of course,

$$
M=\bigoplus_{g \in C} g \otimes U,
$$

where $C$ is a set of coset representatives of $G^{\prime}$ in $G$. Therefore, the annihilator in $K$ of an arbitrary $K$-module composition factor of $M$ must equal $\alpha_{g^{-1}}(Q)$, for some choice of $g \in G$. 
Next, choose an $H$-module composition factor $V$ of $M$, with annihilator $P$ in $H$. It follows from the last paragraph that $P$ lies over $\alpha_{g^{-1}}(Q)$, for some $g \in G$, and hence $\alpha_{g}(P)$ lies over $Q$. Since $V$ was chosen arbitrarily, it now follows from (2.6) that there exist automorphisms $\beta_{1}, \ldots, \beta_{t}$ of $H$ for which

$$
{ }^{\beta_{1}} V, \ldots,{ }^{\beta_{t}} V
$$

is a complete list of the $H$-module composition factors of $M$. In particular, $\operatorname{dim} V$ divides $\operatorname{dim} M=\left[G: G^{\prime}\right] \operatorname{dim} U$. Of course, each finite dimensional simple $H$-module is a composition factor of some $H \otimes_{K} U$, for an appropriate choice of $U$.

The preceding conclusions can all be derived as direct consequences of standard Clifford Theory. In particular, these conclusions can be proved using, for instance, the arguments in $[3,49.2,51.7]$. (Thanks to R. Guralnick and D. Passman for their helpful comments.)

2.8 Lemma. Assume that $H$ is finitely generated as a right $K$-module and that every finite dimensional left $H$-module is semisimple. Let $Q$ be a finite codimensional primitive ideal of $K$, and suppose that $P$ is the annihilator in $H$ of some composition factor of the finite dimensional left $H$-module $H \otimes_{K}(K / Q)$. Then $P$ lies over $Q$.

Proof. Set $M=H \otimes_{K}(K / Q)$, and let $e$ denote the image of $1 \otimes 1$ in $M$. Since $M$ is semisimple as a left $H$-module, there exists a simple left $H$-module factor (i.e., image) $N$ of $M$ with left annihiltor $P$. Consequently, $M / P M$ is a nonzero $H-K$-bimodule factor of $M$, and $M / P M$ must have left annihilator $P$ and right annihilator $Q$. But $Q . e=e . Q=0$, and so $Q$ is the annihilator of a left $K$-submodule of $M / P M$. Hence, $Q$ is minimal over $P \cap K$.

2.9 Proposition. Assume that $H$ is finitely generated as a right $K$-module, that every finite dimensional left $H$-module is semisimple, and that $U$ is a finite dimensional simple left $K$-module.

(i) Let $V_{1}, \ldots, V_{s}$ be the $H$-module composition factors of $H \otimes_{K} U$. Then there exist $k$-algebra homomorphisms $\chi_{2}, \ldots, \chi_{s}: H \rightarrow k$ such that

$$
V_{1} \cong k_{\chi_{2}} \otimes V_{2} \cong \ldots \cong k_{\chi_{s}} \otimes V_{s} .
$$

Consequently, $\operatorname{dim} V_{1}=\operatorname{dim} V_{2}=\cdots=\operatorname{dim} V_{s}$.

(ii) Suppose that $H$ is a free right $K$-module of rank $m$ and that $V$ is an $H$-module composition factor of $H \otimes_{K} U$. Then $\operatorname{dim} V$ divides $m \operatorname{dim} U$.

Proof. (i) Let $P_{1}, \ldots, P_{s}$ be the respective annihilators in $H$ of $V_{1}, \ldots, V_{s}$. Then each $P_{i}$ is the annihilator of some composition factor of $H \otimes_{K}(K / Q)$, where $Q$ is the annihilator in $K$ of $U$. By (2.8), $P_{1}, \ldots, P_{s}$ all lie over $Q$. Part (i) now follows from (2.6).

(ii) Follows easily from (i). 


\section{Proof of Theorem}

This section is a proof of Theorem 1.3 Assume throughout that $H$ is a finite dimensional semisimple $k$-Hopf algebra and that there exists a series of Hopf subalgebras

$$
k=H_{0} \subset H_{1} \subset \cdots H_{t-1} \subset H_{t}=H,
$$

such that $H_{i-1}$ is normal in $H_{i}$, and such that $H_{i} / H_{i} H_{i-1}^{+}$is commutative for all $1 \leq i \leq t$.

It follows from the Nichols-Zoeller theorem (see, e.g., $[10,3.1 .5]$ ) that $H_{j}$ is free as a left and right $H_{i}$-module, for all $1 \leq i \leq j \leq t$. It follows from $[10,3.2 .3]$ that $H_{0}, H_{1}, \ldots, H_{t}$ are all semisimple.

Now set $K=H_{t-1}$, and assume by induction that $K$ is of Frobenius type. Let $m$ denote the rank of $H$ as a free right $K$-module, and let $n=\operatorname{dim} K$. Note that $H$ and $K$ satisfy all of the hypotheses in all of the lemmas in $\S 2$.

Next, let $V$ be a simple left $H$-module. There exists a simple left $K$-module $U$ such that $V$ is an $H$-module composition factor of $H \otimes_{K} U$. It follows from (2.9ii) that $\operatorname{dim} V$ divides $m \operatorname{dim} U$. By the induction hypothesis $\operatorname{dim} U \operatorname{divides} n$, and so $\operatorname{dim} V \operatorname{divides} m n=\operatorname{dim} H$. The theorem follows.

\section{A Class of Applicable Examples}

In this section we briefly outline a class of examples covered by Theorem 1.3 but not by the earlier results $[4 ; 5 ; 11]$ described in (1.1). Our approach is based on [1] (cf. references cited therein).

4.1. Following [1, $\S 3]$, let $A$ and $B$ be finite dimensional $k$-Hopf algebras, let $\rightarrow: B \otimes A \rightarrow A$ be a weak action (in the sense of $[1,2.1]$ ), and let $\rho: B \rightarrow B \otimes A$ be a weak coaction (in the sense of $[1,2.3])$. For $\sigma: B \otimes B \rightarrow A$ and $\tau: B \rightarrow A \otimes A$ satisfying certain restrictions (detailed in $[1, \S 3.1]$ ), one can construct a Hopf algebra

$$
H=A^{\tau} \#_{\sigma} B ;
$$

see $[1,3.1 .12]$. Retain the preceding notation for the remainder of this section.

4.2. It is further proved in $[1, \S 3]$ that: (i) $H$ is isomorphic as an algebra to the crossed product $A \#_{\sigma} B$. (ii) $A$ can be identified isomorphically with a normal Hopf subalgebra of $H$, and

$$
B \cong H / H A^{+} .
$$


4.3. Further assume, for the remainder, that $k$ has positive characteristic $p$, that $X$ is a finite solvable group whose order is not divisible by $p$, that $Y$ is a finite group whose order is divisible by $p$, that $A=k X$, and that $B=(k Y)^{*}$. (It follows that $H^{*}$ is an abelian extension in the sense of [7].)

(i) Since $A$ and $B$ are semisimple, it follows from (4.2i) and [2] that $H$ is semisimple. Since $B$ is commutative and $X$ is solvable, it follows from (4.2ii) that $H$ is lower solvable. Consequently, Theorem 1.3 applies.

(ii) Since the characteristic of $k$ divides $\operatorname{dim} H$, the approach in [5] and [11] cannot be applied. (In particular, since the characteristic of $k$ divides $\operatorname{dim} B,[11,2.1]$ does not apply.) Also, $H$ is not cosemisimple because $B$ is not cosemisimple (see, e.g., [10, 3.2.3]), and so the approach in [4] cannot be applied.

4.4. More concretely, suppose that $Y$ is a finite group whose order is divisible by $p$, and suppose that $X$ is a solvable subgroup of $Y$ whose order is not divisible by $p$. Then $A$ is a left $B$-module algebra via the action

$$
b \rightarrow a=\sum a_{1}\left\langle b, a_{2}\right\rangle
$$

for $a \in A$ and $b \in B$. Letting $H$ now denote the smash product $A \# B$, we can see as above that Theorem 1.3 applies to $H$ but that $[4,5,11]$ do not.

\section{REFERENCES}

1. N. Andruskiewitsch, Notes on extensions of Hopf algebras, Can. J. Math. 48 (1996), 3-42.

2. R. Blattner and S. Montgomery, Crossed products and Galois extensions of Hopf algebras, Pacific J. Math. 137 (1989), 37-54.

3. C. Curtis and I. Reiner, Representation theory of finite groups and associative algebras, Pure and Applied Mathematics, vol. 11, Interscience (Wiley), New York, 1962.

4. P. Etingof and S. Gelaki, On finite dimensional semisimple and cosemisimple Hopf algebras in positive characteristic.

5. __ Some properties of finite-dimensional semisimple Hopf algebras, Math. Res. Lett. 5 (1998), 191-197.

6. I. Kaplansky, Bialgebras, Lecture Notes in Mathematics, Department of Mathematics, University of Chicago, Chicago, 1975.

7. Y. Kashina, G. Mason, and S. Montgomery, Computing the Frobenius-Schur indicator for abelian extensions of Hopf algebras, J. Algebra 251 (2002), 888-913.

8. E. S. Letzter, Noetherian centralizing Hopf algebra extensions and finite morphisms of quantum groups, Bull. London Math. Soc. 31 (1999), 523-530.

9. unity, Alg. Represent. Theory 6 (2003), 47-70.

10. S. Montgomery, Hopf algebras and their actions on rings, CBMS Regional Conference Series in Mathematics 82, Amer. Math. Soc., Providence, 1993.

11. S. Montgomery and S. Witherspoon, Irreducible representations of crossed products, J. Pure Appl. Algebra 129 (1998), 315-326.

12. D. J. S. Robinson, A course in the theory of groups, Second edition, Graduate Texts in Mathematics 80, Springer, New Yourk, 1996.

Department of Mathematics, Temple University, Philadelphia, PA 19122 